\title{
The future of nuclear medicine imaging of neuroendocrine tumors: on a clear day one might see forever...
}

\author{
Lisa Bodei • Mark Kidd • Vikas Prasad • \\ Richard P. Baum • Ignat Drozdov • Irvin M. Modlin
}

Published online: 22 July 2014

(C) Springer-Verlag Berlin Heidelberg 2014

\section{Introduction}

Early identification of neuroendocrine tumors (NETs) is a critical prerequisite to establishing effective treatment. While substantial advances have occurred in the last two decades, there is little progress regarding the identification of small subcentimeter lesions and the determination of tumor proliferative rates and metabolic characteristics. At this time, delineation of lesions mainly utilizes various combinations of somatostatin receptor (SSR) density, glucose metabolism and Hounsfield units.

This editorial addresses unmet needs in nuclear medicine (molecular) imaging with a view to identifying areas that require amplification. The principal goal is to amplify and extend the diagnostic and prognostic role of imaging. Specific focus is required to validate and standardize current techniques while introducing strategies that will resolve currently unmet needs.

\section{Bodei}

Division of Nuclear Medicine, European Institute of Oncology, Milan, Italy

L. Bodei $\cdot$ M. Kidd $\cdot$ I. M. Modlin $(\bowtie)$

Department of Gastroenterological Surgery, Yale School of

Medicine, New Haven, CT, USA

e-mail: imodlin@optonline.net

\section{Prasad}

Department of Nuclear Medicine, Campus Virchow-Klinikum, Charité University Hospital, Berlin, Germany

\section{R. P. Baum}

THERANOSTICS Center for Molecular Radiotherapy and Molecular Imaging (PET/CT), ENETS Center of Excellence, Zentralklinik Bad Berka, Bad Berka, Germany

I. Drozdov

Bering Limited, Richmond, UK

\section{The current status of nuclear medicine imaging of neuroendocrine tumors}

A correct and timely diagnosis is crucial in NET management to establish the most effective treatment [1]. Nuclear medicine techniques, particularly positron emission tomography (PET) scan/ computed tomography (CT), exhibit optimal diagnostic sensitivity for primary and metastatic gastro-enteropancreatic (GEP) NETs. They are usually combined with anatomic techniques to maximize the acquisition of clinically relevant spatial information [2]. In broader terms, a combination of morphological and molecular imaging with radiolabeled probes enables characterization of a lesion in terms of its structural location, anatomical relation, disease extent and its functional status. Overall, the role of nuclear medicine imaging is diagnosis of primary and secondary lesions, assessment of the efficacy of therapy, and to provide information regarding the metabolic and secretory properties of the neoplasia. However, it is evident from published data that there exists a wide variation in diagnostic accuracy. At this time, the most accurate and reproducible (robust) methodology in terms of technology and optimal radioisotope selection is undefined.

\section{Integral components of the techniques}

Nuclear medicine imaging consists of conventional scintigraphy and PET/CT. Scintigraphy is classically carried out with ${ }^{111}$ In-pentetreotide (or OctreoScan ${ }^{\circledR}$ ), or, more rarely, with ${ }^{99 \mathrm{~m}}$ Tc-labeled peptides, like ${ }^{99 \mathrm{~m}} \mathrm{Tc}-\mathrm{HYNIC}-\mathrm{TOC}$, while PET techniques utilize ${ }^{68} \mathrm{Ga}$-DOTA-peptides (DOTA-NOC, -TOC and -TATE - $\left.{ }^{68} \mathrm{Ga}-\mathrm{SMS}-\mathrm{R}-\mathrm{PET}\right)$, and the amine precursors ${ }^{18} \mathrm{~F}-\mathrm{DOPA}$, and ${ }^{11} \mathrm{C}-5$-hydroxytryptophan $\left({ }^{11} \mathrm{C}-5 \mathrm{HTP}\right)$ [3-6].

In nuclear medicine, radiolabeled somatostatin analogs (SSA) are the most utilized, since they represent an optimal 
paradigm of theranostics [7]. Currently, ${ }^{68} \mathrm{Ga}$-SMS-R-PET is regarded as the ideal nuclear medicine NET imaging tool and has, for all practical purposes, displaced OctreoScan ${ }^{\circledR}$. Alternative PET techniques using ${ }^{18} \mathrm{~F}$-DOPA and ${ }^{11} \mathrm{C}-5 \mathrm{HTP}$ are effective, but have been supplanted by the increased availability and better performance of ${ }^{68} \mathrm{Ga}$-SMS-R-PET $[8,9]$.

\section{Strength of each technique}

NET functional imaging comprises a set of tools that facilitates tumor characterization by localization, staging and restaging of both primary and metastatic tumors. In addition, it provides lesion characterization (SSR density, indirect quantification of proliferative activity and tumor heterogeneity through the measurement of glucose metabolism and assessment of substrate metabolism), as well as guiding the selection of therapy (cold or radiolabeled SSAs).

A key clinical impact of functional imaging is provided by its ability to modify therapeutic strategy and prognosis. Thus, somatostatin receptor imaging (SRI), particularly with ${ }^{68} \mathrm{Ga}-$ SMS-R-PET, alters management in $>50 \%$ and successfully predicts response to cold or radiolabeled analogues [10,11]. Although conventional ${ }^{18}$ fludeoxyglucose (FDG) PET is not a primary diagnostic tool in NETs, standardized uptake value (SUV) assessment can provide predictive information in terms of progression-free survival and response to peptide receptor radiotherapy (PRRT) $[12,13]$.

\section{Limitations of nuclear medicine techniques}

Despite the efficacy of ${ }^{68} \mathrm{Ga}$-SMS-R-PET, it is as yet not completely integrated into clinical guidelines [14]. Similarly, functional imaging has not been incorporated into response assessment criteria, which currently rely solely on anatomic information [15]. Despite the high level of awareness of clinicians, the lack of homogeneity regarding the techniques has dampened initial enthusiasm, since comparability has become an issue.

Some of the current limitations of NET functional imaging represent complexities relating to regulatory aspects of isotope/carrier usage as well as to standardization issues. From the regulatory perspective, only ${ }^{111}$ In-pentetreotide has U.S. Food and Drug Administration (FDA) / European Medicines Agency (EMA) approval for NET imaging.

Thus, for ${ }^{68} \mathrm{Ga}$-SMS-R-PET, although four peptides are currently in use (DOTATOC, DOTANOC, DOTATATE and HA-DOTATATE), no marketing authorization for Ge/Ga generators or ${ }^{68} \mathrm{Ga}$-DOTA-peptides exists [16]. These peptides are therefore prepared according to the Good Radiopharmacy Practice. Although a European pharmacopeia monograph on
${ }^{68} \mathrm{Ga}$-DOTATOC was implemented in 2013 (http://www. edqm.eu/medias/fichiers/index_english1.pdf), the EANM guidelines only regulate the general aspects of the procedure. Specific items such as acquisition parameters, administered activity and SUV measurement have been delegated to individual centers [16].

Overall, the methodological limitations of functional imaging include the lack of consensus regarding the optimal agents and techniques (numerous radiopharmaceuticals and scanning techniques, viz. SPECT vs. PET, and for PET, the numerous radiopharmaceuticals available). Furthermore, there is a lack of a fully validated comparison between a state-of-the-art OctreoScan ${ }^{\circledR}$ and ${ }^{68}$ Ga-SMS-R-PET, and no full validation of the ${ }^{68} \mathrm{Ga}-\mathrm{SMS}-\mathrm{R}-\mathrm{PET}$ technique.

\section{Areas that require advance}

${ }^{68}$ Ga-SMS-R-PET validation The requirements are threefold and include: the choice of peptide, the type of radiopharmaceutical preparation, and the reproducibility of the PET technique.

1) Preferred peptide: None of the three peptides currently in use demonstrate a clear diagnostic superiority over the others [17, 18].

2) Preparation: Apart from DOTATOC, which has a European Pharmacopeia monograph, radiopharmaceuticals are prepared according to non-standardized local procedures. Furthermore, no standardized toxicity and sterility data exist.

3) PET technique reproducibility: Current protocols are not uniformly consistent in the acquisition time point or the optimal preparation of patients, i.e., the time interval between cold SSA injection and imaging. The semiquantitative parameter to quantify uptake, i.e. the SUV, is intrinsically variable and varies significantly among individual PET scanners and between centers. Moreover, its reproducibility may be altered by the use of different peptides (with different receptor affinities), by different scanning times (resulting in different phases of tumor uptake), by the degree of receptor saturation in normal tissue due to the co-administration of cold analogues [19] and by splenectomy [20].

${ }^{18} \mathrm{FDG}$ uptake Increased ${ }^{18} \mathrm{FDG}$ uptake as a prognostic marker for NET aggression requires rigorous clinical validation. In parallel, its correlation with the tumor Ki67 index, the transcript proliferome or other indices of proliferation have not been adequately delineated. For example, ${ }^{18} \mathrm{FDG}$ is positive in a substantial percentage of slow-proliferating low grade (G1) tumors, confounding the generally accepted notion that it is 
only useful in the identification of rapidly proliferating and poorly differentiated tumors [13].

Biologic information There is a need to integrate the biological information of NET pathophysiology with nuclear medicine diagnostic and therapeutic strategies. Thus, the relationship between the intrinsic variability of individual NET cells (EC, beta, ECL, D, Clara etc.) that comprise the different tumor types and nuclear medicine strategies requires investigation. This should include a delineation of histopathological indices, high-throughput molecular analyses, receptor subtyping and characterization as well as the definition of metabolic parameters that delineate function and proliferation. Aggregation of such information will provide added value in the interpretation of diagnostic scans and further inform the efficacy of therapeutic strategies.

\section{The future}

1) Strategies to advance current techniques

The development of an efficient and reproducible SSR-based molecular imaging procedure is a strategic necessity to ensure clinical acceptance of current and future techniques. The recent assignment by FDA/EMA of orphan drug designation to ${ }^{68} \mathrm{Ga}$-DOTATOC and ${ }^{68} \mathrm{Ga}-$ DOTATATE is, hopefully, a step towards uniformity.

A critical issue is the need to validate ${ }^{68} \mathrm{Ga}$-SMS-RPET, especially by assuring SUV objectivity and comparability. Options include correction of the tumor maximum SUV ( $\left.\mathrm{SUV}_{\max }\right)$ with background [21], the correction of the tumor $\mathrm{SUV}_{\max }$ with the spleen [22], and the calculation of molecular tumor volume [23]. Finally, in order to be predictive, the apocryphal "Rotterdam scale" needs to be objectified and adapted to PET. The collaboration of clinical and nuclear medicine societies in the process of standardization is necessary to assure adoption of a uniform process.

2) Introduction of novel techniques and strategies

Improvements in current techniques that utilize Ga-68 are worthy of consideration. Thus, SSAs labeled with $\mathrm{Cu}-$ 64 are of considerable interest, due to the excellent image quality and the spatial resolution [24]. The 12.5-hour halflife allows later imaging compared to Ga-peptides, with stable tumor to background ratios at least $3 \mathrm{~h}$ after injection, thus matching more closely the tumor uptake kinetics, and the possibility of imaging at $24 \mathrm{~h}$.

An additional area is the development of alternative fusion imagery. Preliminary studies with fused ${ }^{68} \mathrm{Ga}-$ SMS-R-PET and magnetic resonance imaging (MRI) scan, both as diffusion-weighted and gadoxetateenhanced images, have demonstrated similar high per- region (98.9 and $97.7 \%$, respectively) and per-organ (95.7 and $91.3 \%$, respectively) sensitivity, with comparable high specificity (99.6-99.7\%) [25]. The PET and MRI techniques provide complementary information, regarding both the anatomical detail and the functional characterization of the tissue, including the diffusionweighted imaging (DWI) parameters and the receptormediated uptake of the ${ }^{68} \mathrm{Ga}$-DOTA-peptide.

SSAs have been the workhorse of imagery for two decades, and alternative radiopharmaceuticals that provide increased diagnostic accuracy should be identified. In this respect, the use of SSR antagonists appears to represent a highly promising strategy [26]. The lack of internalization and the recognition of increased binding sites represent an inversion of the current paradigm of agonists. Agents such as ${ }^{111}$ In-DOTA-BASS $\left[{ }^{177} \mathrm{Lu}-\right.$ DOTA-p $\mathrm{NO}_{2}$-Phe-c (dCys-Tyr-dTrp-Lys-Thr-Cys) $\mathrm{dTyrNH}_{2}$ ] or ${ }^{111}$ In-DOTA-JR11 (DOTA-Cpa-c[D-CysAph(Hor)-D-Aph(Cbm)-Lys-Thr-Cys]-D-Tyr-NH2) exhibit higher levels and longer retention rates, thereby providing a higher sensitivity compared to ${ }^{111}$ Inpentetreotide [27]. In terms of therapeutic application, in vitro studies indicate a significantly greater binding of ${ }^{177} \mathrm{Lu}$-DOTA-BASS on neuroendocrine tumor cells than the current best agonist ${ }^{177} \mathrm{Lu}$-DOTATATE [28]. The clinical translation of this observation suggests a higher tumor accumulation with increased irradiation, while the lower normal tissue accumulation implies diminished exposure [29]. Of note, however, is the observation that the somatostatin receptor affinity of such compounds can be diminished by binding to radiometals, such as Ga-68. To retain optimal SSTr binding of the JR11 antagonist, a specific 1,4,7-triazacyclononane,1-glutaric acid-4,7acetic acid (NODAGA) chelator was required [27].

The development of novel receptor targets utilizing innovative radiopharmaceuticals needs identification. In particular, specific agents that identify a particular tumor or one with a specific secretory product need to be investigated. A number of peptides have been tested in preclinical and clinical trials. Among these, the GLP-1 receptor (GLP-R) peptides, such as ${ }^{111}$ In-exendin-4 (localization of occult insulinomas), are the most advanced for clinical application [30]. ${ }^{111}$ In-exendin-4 specifically addresses the paucity of SSRs in benign insulinomas. In this respect, GLP-R and SSR imaging demonstrate the biologically mutable aspect of insulinomas, which may be GLP-R positive and SSR negative or vice-versa, a reflection of their malignant phenotype [31]. A ${ }^{68} \mathrm{Ga}$-labeled exendin4 is the logical next step and has been tested in clinical trials $[32,33]$.

Following the same principle of the paradigm shift from agonist to antagonists, it was recently demonstrated that the ${ }^{125}$ I-BH-exendin(9-39) GLP-1 antagonist has 
excellent binding properties and constitutes a promising imaging agent [34].

Similar evaluation of the multi-receptor expression of neuroendocrine cells has demonstrated that the cholecystokinin/gastrin ligands, such as $\mathrm{CCK}_{8}$ and minigastrin analogs labeled with ${ }^{111} \mathrm{In}$ or ${ }^{99 \mathrm{~m}} \mathrm{Tc}$, may also have clinical utility [35].

Other targets that have been explored for NET imaging include the bombesin receptor family, which include GRP (gastrin-releasing peptide), NMB (neuromedin B), and $\mathrm{BB}_{3}$ (bombesin receptor subtype 3) receptors [36]. In vitro usage to identify prostate cancer indicates positive identification in 60-100\% [35-39]. Similarly, GRP and $\mathrm{BB}_{3}$ receptors have been identified in $7 / 10$ and $2 / 10$ gastrinomas, respectively, NMB receptors have been found in 11/27 ileal, while BB3 receptors were the predominant receptors described in 10/29 bronchial NETs [36]. More than 40 different bombesin analogs, agonists and antagonists labeled with ${ }^{99 \mathrm{~m}} \mathrm{Tc}$ or with ${ }^{68} \mathrm{Ga}$ have been evaluated in vitro. They comprise an additional potential class of radiopharmaceuticals for NET imaging [37].

The low plasma stability and high kidney retention have limited the application of these alternative peptides as theranostics [40]. Nevertheless, newer, more stable molecules and the co-administration of specific enzyme inhibitors, such as the neutral endopeptidase inhibitor phosphoramidon, can be utilized to increase the bioavailability of these compounds [41]. Adjunctive strategies of this type are likely to herald a new era in the application of receptor peptides and supplant the model of somatostatin analogs in the study of neuroendocrine tumors.

Assessment of alternative components of NET biology such as angiogenesis has led to the development of promising strategies utilizing radiolabeled antibodies such as the Zr-89-labeled bevacizumab. This concept has been applied to the evaluation of a variety of NETs treated with everolimus. A decrease in the SUV led to the proposal that this technique could be of value as an early predictor of anti-angiogenetic therapeutic efficacy [42].

3) Utilization of other biologic information to amplify accuracy

It is clear that monoanalyte-derived information can never be as effective as the product of multianalyte parameters. Thus, an image alone is, by definition, limited only by the lack of additional, relevant parameters that can be integrated into an amplifiable diagnostic quotient. Inclusion of such additional material, in a mathematical probability index, or in a matrix or via a nomogram has proved of considerable added prognostic value in other disciplines [43]. The multi-level parallel assessment of different forms of tumor/patient relevant information is mandatory to strengthen diagnostic and prognostic accuracy. To optimally increase information gained from nuclear medicine techniques, NET images will likely require integration of tumor and blood biomarkers and the development of prognostic nomograms. A particularly informative source of information would be the integration of circulating tumor genomic data obtained from blood (simultaneous liquid biopsy) at the time of nuclear medicine image acquisition. Thus, receptor determined tumor characteristics could be combined with tumor transcript profiles, allowing for the development of a personalized predictive assessment of tumor status before, during and after treatment [44].

\section{Coda}

The integration of functional and anatomic imaging optimizes the delineation of the status of a NET. PET, particularly with ${ }^{68} \mathrm{Ga}$-DOTA-peptides, is supplanting OctreoScan ${ }^{\circledR}$. Emerging strategies include the use of SSR-antagonists and GLP1-R peptides. However, the present lack of homogeneity and validation has limited the clinical acceptance of novel techniques. A robust and standardized basis to objectify nuclear medicine procedures is a critical requirement.

The future development of multi-dimensional-algorithmic data quotients (tissue, blood and imaging) for each patient, as opposed to a mono-dimensional image-based procedure, is likely to generate information that is far more accurate than the current strategy. In this respect, the combination of a simultaneous gene transcript blood signature from the tumor as well as a functional image may provide an informative mechanism for capturing knowledge of both the biology of an individual tumor, as well as its current and future behavior.

\section{References}

1. Modlin IM et al. Gastroenteropancreatic neuroendocrine tumours. Lancet Oncol. 2008;9(1):61-72.

2. Sundin A. Radiological and nuclear medicine imaging of gastroenteropancreatic neuroendocrine tumours. Best Pract Res Clin Gastroenterol. 2012;26(6):803-18. doi:10.1016/j.bpg.2012.12. 004.

3. Bodei L et al. Peptide receptor therapies in neuroendocrine tumors. J Endocrinol Investig. 2009;32(4):360-9.

4. Gabriel $\mathrm{M}$ et al. 68Ga-DOTA-Tyr3-Octreotide PET in neuroendocrine tumors: comparison with somatostatin receptor scintigraphy and CT. J Nucl Med. 2007;48(4):508-18.

5. Koopmans KP et al. Improved staging of patients with carcinoid and islet cell tumors with 18 F-dihydroxy-phenyl-alanine and 11C-5- 
hydroxy-tryptophan positron emission tomography. J Clin Oncol. 2008;26(9):1489-95.

6. Orlefors $\mathrm{H}$ et al. Whole-body (11)C-5-hydroxytryptophan positron emission tomography as a universal imaging technique for neuroendocrine tumors: comparison with somatostatin receptor scintigraphy and computed tomography. J Clin Endocrinol Metab. 2005;90(6):3392-400.

7. Baum RP, Kulkarni HR, Carreras C. Peptides and receptors in imageguided therapy: theranostics for neuroendocrine neoplasms. Semin Nucl Med. 2012;42(3):190-207. doi:10.1053/j.semnuclmed.2012. 01.002 .

8. Ambrosini V et al. Comparison between 68Ga-DOTA-NOC and 18F-DOPA PET for the detection of gastro-entero-pancreatic and lung neuro-endocrine tumours. Eur J Nucl Med Mol Imaging. 2008;35(8):1431-8. Epub 2008 Apr 17.

9. Haug A et al. Intraindividual comparison of 68Ga-DOTA-TATE and 18F-DOPA PET in patients with well-differentiated metastatic neuroendocrine tumours. Eur J Nucl Med Mol Imaging. 2009;36(5): 765-70. Epub 2009 Jan 10.

10. Ambrosini $\mathrm{V}$ et al. 68Ga-DOTANOC PET/CT clinical impact in patients with neuroendocrine tumors. J Nucl Med. 2010;51(5):669 73. Epub 2010 Apr 15.

11. Campana D et al. Standardized uptake values of (68)Ga-DOTANOC PET: a promising prognostic tool in neuroendocrine tumors. J Nucl Med. 2010;51(3):353-9. Epub 2010 Feb 11.

12. Binderup T et al. 18F-fluorodeoxyglucose positron emission tomography predicts survival of patients with neuroendocrine tumors. Clin Cancer Res. 2010;16(3):978-85. Epub 2010 Jan 26.

13. Severi $\mathrm{S}$ et al. Role of $18 \mathrm{FDG} \mathrm{PET} / \mathrm{CT}$ in patients treated with 177Lu-DOTATATE for advanced differentiated neuroendocrine tumours. Eur J Nucl Med Mol Imaging. 2013;40(6):881-8. doi:10. 1007/s00259-013-2369-z. Epub 2013 Feb 27.

14. Kwekkeboom DJ et al. ENETS consensus guidelines for the standards of care in neuroendocrine tumors: peptide receptor radionuclide therapy with radiolabeled somatostatin analogs. Neuroendocrinology. 2009;90(2):220-6.

15. Eisenhauer EA et al. New response evaluation criteria in solid tumours: revised RECIST guideline (version 1.1). Eur J Cancer. 2009;45(2):228-47.

16. Virgolini I et al. Procedure guidelines for PET/CT tumour imaging with 68Ga-DOTA-conjugated peptides: 68Ga-DOTA-TOC, 68GaDOTA-NOC, 68Ga-DOTA-TATE. Eur J Nucl Med Mol Imaging. 2010;37(10):2004-10.

17. Poeppel TD et al. 68Ga-DOTATOC versus 68Ga-DOTATATE PET/ $\mathrm{CT}$ in functional imaging of neuroendocrine tumors. J Nucl Med. 2011;52(12):1864-70. Epub 2011 Nov 9.

18. Kabasakal L et al. Comparison of (6)(8)Ga-DOTATATE and (6)(8)Ga-DOTANOC PET/CT imaging in the same patient group with neuroendocrine tumours. Eur J Nucl Med Mol Imaging. 2012;39(8):1271-7. Epub 2012 Apr 20.

19. Sabet A et al. Does PRRT with standard activities of $177 \mathrm{Lu}-$ octreotate really achieve relevant somatostatin receptor saturation in target tumor lesions?: insights from intra-therapeutic receptor imaging in patients with metastatic gastroenteropancreatic neuroendocrine tumors. EJNMMI Res. 2013;3(1):82. doi:10.1186/2191-219X-3-82.

20. Kratochwil $\mathrm{C}$ et al. Comparison of $68 \mathrm{Ga}$-DOTATOC biodistribution in patients with and without spleenectomy. Q J Nucl Med Mol Imaging. 2014;2:2.

21. Garin E et al. Predictive value of 18F-FDG PET and somatostatin receptor scintigraphy in patients with metastatic endocrine tumors. J Nucl Med. 2009;50(6):858-64. Epub 2009 May 14.

22. Haug AR, Auernhammer CJ, Wängler B, Schmidt GP, Uebleis C, Göke B, et al. 68Ga-DOTATATE PET/CT for the early prediction of response to somatostatin receptor-mediated radionuclide therapy in patients with well-differentiated neuroendocrine tumors. J Nucl Med. 2010;51(9):1349-56. doi:10.2967/jnumed. 110.075002 .
23. Baum RP, Kulkarni HR. THERANOSTICS: from molecular imaging using Ga-68 labeled tracers and PET/CT to personalized radionuclide therapy - the Bad Berka experience. Theranostics. 2012;2(5):43747. doi:10.7150/thno.3645. Epub 2012 May 7.

24. Pfeifer $\mathrm{A}$ et al. Clinical PET of neuroendocrine tumors using $64 \mathrm{Cu}-$ DOTATATE: first-in-humans study. J Nucl Med. 2012;53(8):120715. doi:10.2967/jnumed.111.101469. Epub 2012 Jul 10.

25. Mayerhoefer ME et al. Are contrast media required for (68)GaDOTATOC PET/CT in patients with neuroendocrine tumours of the abdomen? Eur Radiol. 2012;22(4):938-46. Epub 2011 Nov 12.

26. Waser $B$ et al. Highly efficient in vivo agonist-induced internalization of sst2 receptors in somatostatin target tissues. J Nucl Med. 2009;50(6):936-41. doi:10.2967/jnumed.108.061457. Epub 2009 May 14.

27. Fani M, Braun F, Waser B, Beetschen K, Cescato R, Erchegyi J, et al. Unexpected sensitivity of sst 2 antagonists to N-terminal radiometal modifications. J Nucl Med. 2012;53(9):1481-9.

28. Cescato $\mathrm{R}$ et al. Evaluation of 177Lu-DOTA-sst2 antagonist versus 177Lu-DOTA-sst2 agonist binding in human cancers in vitro. J Nucl Med. 2011;52(12):1886-90.

29. Wild D et al. First clinical evidence that imaging with somatostatin receptor antagonists is feasible. J Nucl Med. 2011;52(9):1412-7. doi: 10.2967/jnumed.111.088922. Epub 2011 Aug 18.

30. Wild D et al. Glucagon-like peptide 1-receptor scans to localize occult insulinomas. N Engl J Med. 2008;359(7):766-8.

31. Wild D, Christ E, Caplin ME, Kurzawinski TR, Forrer F, Brändle M, et al. Glucagon-like peptide-1 versus somatostatin receptor targeting reveals 2 distinct forms of malignant insulinomas. J Nucl Med. 2011;52(7):1073-8. doi:10.2967/jnumed.110.085142.

32. Selvaraju RK et al. In vivo imaging of the glucagonlike peptide 1 receptor in the pancreas with $68 \mathrm{Ga}$-labeled DO3A-exendin-4. J Nucl Med. 2013;54(8):1458-63. doi:10.2967/jnumed.112.114066. Epub 2013 Jun 12.

33. Eriksson $\mathrm{O}$ et al. Detection of metastatic insulinoma by positron emission tomography with [(68)ga]exendin-4-a case report. J Clin Endocrinol Metab. 2014;99(5):1519-24.

34. Waser B, Reubi JC. Radiolabelled GLP-1 receptor antagonist binds to GLP-1 receptor-expressing human tissues. Eur J Nucl Med Mol Imaging. 2014;41(6):1166-71.

35. Fani M, Maecke HR. Radiopharmaceutical development of radiolabelled peptides. Eur J Nucl Med Mol Imaging. 2012;39 Suppl 1:S11-30.

36. Reubi JC, Waser B. Concomitant expression of several peptide receptors in neuroendocrine tumours: molecular basis for in vivo multireceptor tumour targeting. Eur J Nucl Med Mol Imaging. 2003;30(5):781-93.

37. Sancho V, Di Florio A, Moody TW, Jensen RT. Bombesin receptormediated imaging and cytotoxicity: review and current status. Curr Drug Deliv. 2011;8(1):79-134.

38. Cescato $\mathrm{R}$ et al. Bombesin receptor antagonists may be preferable to agonists for tumor targeting. J Nucl Med. 2008;49(2):318-26.

39. Reubi JC. Old and new peptide receptor targets in cancer: future directions. Recent Results Cancer Res. 2013;194:567-76.

40. Ambrosini V et al. Radiopeptide imaging and therapy in Europe. $\mathrm{J}$ Nucl Med. 2011;52 Suppl 2:42S-55.

41. Nock BA et al. "To serve and protect": enzyme inhibitors as radiopeptide escorts promote tumor targeting. J Nucl Med. 2014;55(1):121-7.

42. van Asselt SJ et al. Everolimus reduces $89 \mathrm{Zr}$-Bevacizumab tumor uptake in patients with neuroendocrine tumors. J Nucl Med 2014.

43. Modlin IM et al. A nomogram to assess small-intestinal neuroendocrine tumor ('carcinoid') survival. Neuroendocrinology. 2010;92(3): 143-57. Epub 2010 Aug 23.

44. Modlin I, Drozdov I, Kidd M. The identification of gut neuroendocrine tumor disease by multiple synchronous transcript analysis in blood. Plos One 2013. e63364. 